\title{
NANOSCALE SCIENCE AND TECHNOLOGY FOR THE DEVELOPMENT OF ENVIRONMENTAL SENSORS
}

\author{
Grant \# DE-FG02-01ER15207
}

\author{
Final Report \\ Jaunary, 2007
}

\author{
Faculty: \\ Ronald Andres, School of Chemical Engineering, Purdue University \\ David Janes, School of Electrical and Computer Engineering, Purdue University \\ Clifford Kubiak, Dept. of Chemistry, UCSD \\ Ronald Reifenberger, Dept. of Physics, Purdue University
}

\section{Graduate Students:}

Venugopal Santhanam, Rajan Agarwal, School of Chemical Engineering, Purdue University

Gabor Pszota, Physics, Purdue University

Jaewon Choi, Electrical and Computer Engineering, Purdue University

Scott Burns, Department of Chemistry, UCSD

Degrees: Venugopal Santhanam, PhD Purdue University, December 2002, "Fabrication of Nanoelectronic Devices Using Self-Assembled 2D Arrays of Monolayer Protected Clusters", Jaewon Choi, "Development of Step Junction Devices“, Aug. 2004.

\section{Publications:}

1. "Self-assembly of uniform monolayer arrays of nanoparticles," V. Santhanam, J. Liu, R. Agarwal, R.P. Andres, Langmuir, 19, 7881 (2003).

2. "Microcontact Printing of uniform nanoparticle arrays," V. Santhanam and R.P. Andres, Nano Lett., 4, 41 (2004).

3. J. Choi, D. B. Janes, S. Lodha, Y. Chen, R. Agarwal, R. P. Andres, S. Burns, C. P. Kubiak, "Conduction Through Molecule-Gold Cluster Complexes and Applications," Proc. of 2003 IEEE Nanotechnology Conference, San Francisco, CA, Aug. 2003.

4. J. Choi, K. Lee and D. B. Janes, "Nanometer Scale Gap made by Conventional Micro-scale Fabrication," Nanoletters, Vol. 4, p. 1699-1703, 2004.

5. "Time Evolution Studies of the Electrostatic Surface Potential of Low Temperature Grown GaAs Using Electrostatic Force Microscopy", S. Howell and D. B. Janes, to appear in Journal of Applied Physics, 2005. 


\section{Patents}

Patent Application: R.P. Andres, V. Santhanam, R. Agarwal, "Fabrication of Nanoparticle Arrays," Purdue Research Foundation, August 2004.

\section{Invited Conference Talks and Presentations}

"Metal Nanoparticles and Their Self-Assembly into Electronic Nanostructures", R. P. Andres, Invited Lecture March 25, 2004, Department of Chemical and Biological Engineering, Johns Hopkins University, Baltimore, MD.

"Molecular Heterostructures for Electronic Devices,“ D. B. Janes, USA-Japan Workshop on Molecular Electronics, Chandler, AZ, March 2002.

"Integrating Molecular Elements into Macroscale Components," D. B. Janes, 2002 AICHE Meeting (American Institute of Chemical Engineers) (Plenary session in Nanotechnology), Indianapolis, Indiana, Nov. 4-8, 2002.

"Metal-Molecule-Metal Structures with Pre-Fabricated Contacts, D. B. Janes, S. Ghosh, H. Halimun, J. Choi, S. Burns and C. P. Kubiak, $6^{\text {th }}$ Engineering Foundation Conference on Molecular-Scale Electronics, Key West, FL, Dec. 15-19, 2002.

"Metal-Molecule-Metal and Metal-Molecule-Semiconductor Devices," D. B. Janes, Annual Nanotechnology Center Conference, University of South Carolina, April, 2003.

"Circuit Applications of Molecular Electronics Components," D. B. Janes, IEEE Application Specific Architecture and Processing (ASAP) Conference, The Hague, Netherlands, June 2003.

"Molecular Devices with Pre-Formed Contacts," D. B. Janes, 1st Annual Workshop on Molecular Conduction, W. Lafayette, IN, July, 2003.

"Metal-Molecule-Metal and Metal-Molecule-Semiconductor Devices," D. B. Janes, USFrance Workshop on Molecular Electronics, Paris, France, Dec. 17-19, 2003.

"Metal-Molecule-Metal and Metal-Molecule-Semiconductor Devices," D. B. Janes, S. Ghosh, S. Lodha, J. Choi and S. Bhattacharya, IEEE Nanoscale Devices and Systems Integration Conference, Miami, FL, Feb. 16-19, 2004.

"Chemically Gating Conduction in Molecular Nanostructures", C. P. Kubiak, invited lecture, Trends in Nanotechnology 2001, Segovia, Spain, September 3-7, 2001.

“Electronic Conduction in Molecular Nanostructures”, C. P. Kubiak, invited lecture, 
Inaugural Donald D. Harrington Symposium on Nanoscience, University of

Texas Austin, November 2, 2001.

"Electronic Conduction in Molecular Nanostructures", C. P. Kubiak, invited colloquium, Mississippi State University, December 3, 2001.

"Electronic Conduction in Molecular Nanostructures", C. P. Kubiak, invited seminar, University of Colorado - Boulder, January 29, 2002.

"Electronic Conduction in Molecular Nanostructures", C. P. Kubiak, invited lecture, Baekeland Award Symposium, Rutgers University, May 24, 2002.

"Electronic Conduction in Molecular Nanostructures. Chemical Control of the Current-Voltage Response", C. P. Kubiak, invited lecture, Gordon Research Conference on Electron Donor Acceptor Interactions, Newport, R. I., August 11-15, 2002.

(1) "Infrared Vibrational Lineshape Coalescence in Inorganic Charge Transfer Complexes: It Looks like Dymanic NMR Running at One Billion X, But is it?"; (2) Electronic Conduction in Molecular Nanostructures. Chemical Control of the Current-Voltage Response"; (3) Atom Transfer Chemistry of Organometallic Complexes: Hydrocarbation of Alkenes by Nickel Carbenes", C. P. Kubiak, Distinguished Lectures in Inorganic Chemistry, Northwestern University, September 9 11, 2002.

"Molecular Based Chemical Sensors", C. P. Kubiak, invited lecture, NASA Institute for Nanoelectronics and Computing Kickoff Meeting, Purdue University, January 16-17, 2003.

"Molecular Electronics and Nanostructures", C. P. Kubiak, Haines Distinguished Lecture, University of South Dakota, April 14, 2003.

(1) "Chemical Nanosensors"; (2) "Molecular Electronics and Nanostructures", C. P. Kubiak, invited lectures, NSF Workshop on Molecular Electronics and Quantum Dots; Quilmes, Argentina, May 15-20, 2003.

"Conducting molecular nanostructures assembled from charge-transfer complexes grafted onto silicon surfaces", C. P. Kubiak, invited plenary lecture, SPIE Meeting, San Diego, CA, August 3 8, 2003.

'Chemical Control Of Conductance: "Doping" Molecules, ChemResistors, and Chem-FETs', C. P. Kubiak, Invited plenary lecture, Trends in Nanotechnology 2003, Salamanca, Spain, September 15-19, 2003. 


\section{Summary of Technical Accomplishments}

Under this funding, we proposed to: i) develop a ChemFET sensor platform, ii) develop a ChemDiode sensor platform, iii) synthesize receptor molecules suitable for chemical sensing, iv) study the electrostatic potential changes induced by receptor/target binding on surfaces and v) develop VLSI fabrication approaches for micron-scale chemical sensor devices. The accomplishments under these various thrusts are summarized in this section.

\section{Fabrication of ChemFET Sensor Platform}

We are developing integrated devices that combine sensor molecules with semiconductor device structures to provide sensitive, selective, chemical detection on a platform that interfaces seamlessly to solid-state electronic circuitry for signal amplification, logic, and transmission. FETs operate via application of a threshold voltage $\left(V_{T}\right)$ to the gate electrode to induce the formation of a channel to assist or deter the drift of electrons between source and drain electrodes to turn on or off the device, Figure 2. In the proposed work it is possible to form a channel between source and drain electrodes of a FET using a chemical binding event. A SAM with specific chemical binding affinities is chemisorbed onto a thin layer of gold deposited over the gate. The sensor molecule is chosen to specifically bind a target molecule in a way that produces a substantial change in dipole moment after binding. The change in a sheet of dipoles acts as an applied voltage which turns on the FET. Although the magnitude of the dipole change due to a chemical binding event may not be strong enough to induce a change in state of the FET on its own (e.g. going from OFF $\rightarrow \mathrm{ON}$ ), it is possible to bias the system via back gating at or near the $V_{T}$ such that a small dipole change will result in a change in state of the FET.

Fabrication of a suitable MOSFET is necessary to meet the needs of the low biased gate enhancement brought on by chemical binding. The voltage threshold of the FET is directly related to the fabrication process and ultimately the lower the voltage threshold the more sensitive the device. Although functionalization of a gold gate electrode will alter the work function and shift the $V_{T}$, careful characterization of the FET by clearly defining the $V_{T}$ for the unfunctionalized FET will allow for an approximation of the magnitude of back gating required to bring the FET near the $V_{T}$. Optimization of the type and thickness of the insulating layer as well as the length, width, and thickness of the gate electrode can be used to modify the response of the FET. Careful characterization of the FET will be necessary to optimize the response of the sensor to its target molecule.

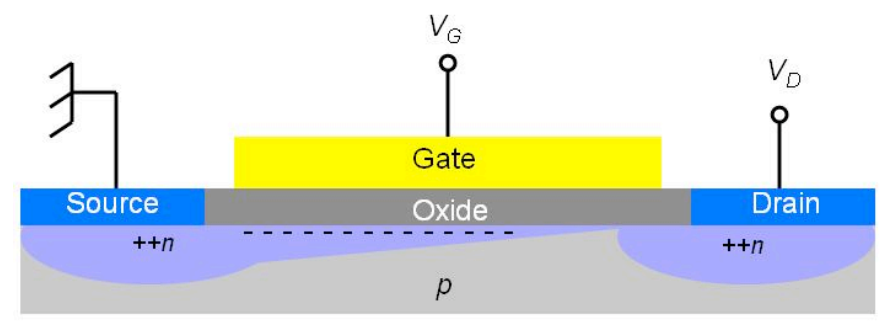

Figure 2. Field-Effect transistor (FET) structure developed for chemical sensing. For sensing, the effective gate potential is induced by charge dipoles associated with binding to sensor molecules on the gate electrode.

Development of a ChemFET platform for multiple use chemical sensing can be facilitated by taking advantage of the specificity of known chemical binding such as the formation of self-assembled monolayers of thiol terminated molecules. ${ }^{19,20}$ The resultant build up of charge from the separation in the distance between a polar head group and a non-polar tail on the surface of gold is quantifiable by Kelvin Probe measurements. The measurable surface 
potentials of various SAMs will be used to characterize the response of the FET to SAM formation. Initial work will study the correlation of the drain current $\left(\mathrm{I}_{\mathrm{D}}\right)$ in the FET to the chain length of an alkane thiol bound to a gold gate electrode. This study will also help characterize the FET by probing the minimum gate potential necessary to observe a change in response in the $\mathrm{I}_{\mathrm{D}}$.

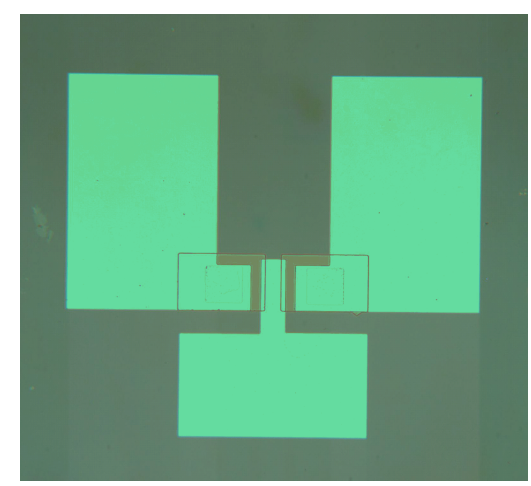

Figure 3. ChemFET platform with $60 \mu \mathrm{m} \times 110 \mu \mathrm{m}$ gate electrode

The proposed ChemFET platform provides amplification of the signal, since the output current of the device shifts by a factor of 10 for each $60 \mathrm{mV}$ change in the gate potential (threshold). Since typical molecular dipole layers on surfaces induce potential shifts of 100-200 $\mathrm{mV}$ (corresponding to 0.1-0.2 Debye per molecule), ChemFET devices should be able to detect events in which target gas molecules bind to less than $0.1 \%$ of the molecules on the gate. For a device with $100 \mathrm{~nm} \times 500 \mathrm{~nm}$ gate dimensions, this would correspond to a sensitivity limit of approximately 50 molecules.

A ChemFET device has been fabricated on a low doped n-type silicon wafer (Fig. 3). Using standard photolithography techniques for registration and fabrication of structures the following steps were used: 1) the source and drain regions were doped with boron, 2) a 1000um gate oxide was grown, 3) $\mathrm{Au}$ was e-beam evaporated to form the source, drain, and gate electrodes. Currently, the FET device has a $60 \mu \mathrm{m}$ $\mathrm{x} 110 \mu \mathrm{m}$ gate. It is undergoing initial testing with SAMs of alkane thiols. Characterization of the SAMs by surface IR, ellipsometry, and contact angle measurements have been completed. Analysis of the FET is being performed to characterize the response of the FET to varying gate potential.

Future work will involve a systematic evaluation of the response of a FET to various complexes. Possible candidates will be screened via Kelvin Probe measurements. Acceptable systems with large enough dipole changes will be adapted for FET sensing. This will allow for the build-up of a library of FET responses to small molecules for the purpose of chemical fingerprint identification.

The standard photolithographic fabrication of the ChemFET allows incorporation of these sensor elements into microelectronic circuits. Integrated circuits can be used to further amplify the sensor signal, identify the presence of specific gases and transmit the results. The ChemFET shown in Fig. 3 is a generic structure. Sensors for various gases can be fabricated on the same chip by incorporating different sensor molecules on individual ChemFETs.

\section{Fabrication of ChemDiode Sensor Platform}

ChemDiode sensors employ conjugated sensor molecules to electronically link $\mathrm{Au}$ nanoparticles in a close-packed bilayer film. The linked nanoparticle bilayer forms the conduction path between source and drain electrodes on a oxidized silicon substrate. A schematic illustration of a ChemDiode is shown in Fig. 4. 

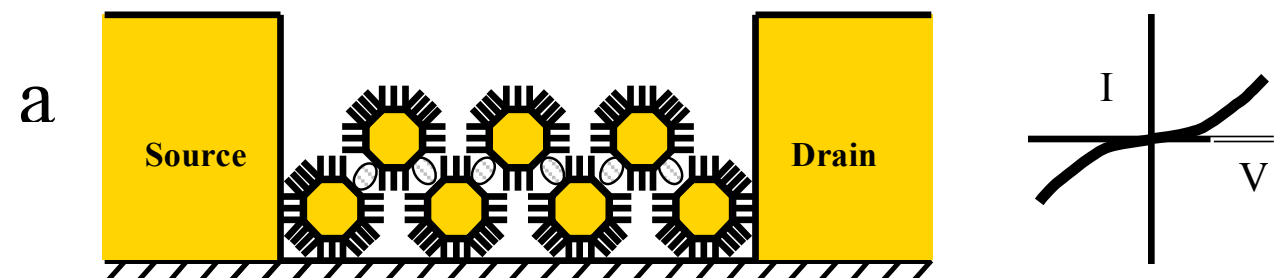

b
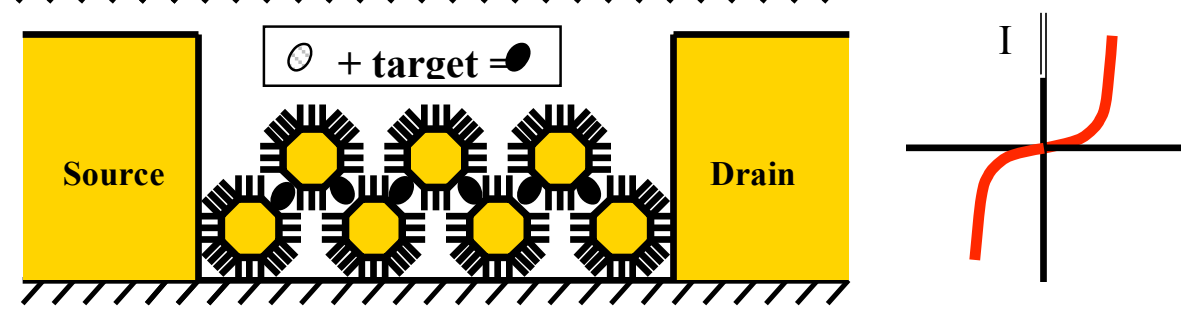

Figure 4: Side view schematic of a ChemDiode device consisting of a close-packed bilayer film of Au nanoparticles connecting source and drain electrodes on an insulating substrate. The Au nanoparticles in the two layers of the bilayer film are linked by sensor molecules whose electrical resistance is sensitive to the presence of a target species. The inserts at the left show the characteristic I(V) behavior of such a ChemDiode (a) before exposure to a targeted ligating molecule and (b) after exposure to a target ligating molecule.

Although the ChemDiode sensor is a chem-resistive device, it is fundamentally different from all current chem-resistive sensors. Current sensors are based on measuring the electrical resistance of relatively thick (i.e. micron-scale not nanometer-scale) inorganic or organic films. The inorganic film based devices are usually made with chemically doped $\mathrm{SnO}_{2}$. Although these devices have good reproducibility and sensitivity, they have relatively high power consumption and poor chemical specificity. Organic film based devices employ composite films containing conductive fillers such as vanadium oxide or carbon black particles embedded in a polymer matrix. The sensing mechanism involves swelling of the polymer matrix due to adsorption of the gas being detected. This causes a change in the relative distance between the filler particles and alters the electron tunneling resistance between the particles. These organic film devices have lower power consumption and better chemical specificity but suffer from slow response times and poor long term stability. Recently, chem-resistive sensors based on sub-micron thick amorphous films of coated metal nanoparticles have been proposed. ${ }^{21-23}$ The mechanism by which these sensors operate is the same as that of organic film devices with the organic molecules encapsulating the metal nanoparticles serving as the matrix which swells on adsorption of gas molecules. These sensors have improved response times, however, their sensitivity and selectivity are relatively poor. At best they can distinguish between polar and non-polar vapors.

The key difference between the ChemDiode sensor and existing chem-resistive sensors is the fact that the electrical response of the ChemDiode is due to the chemical binding of a target species with the sensor element molecules. This response is non-linear (Fig. 4), is target species specific, and can be extremely large (we have recently demonstrated a seventy-fold change in conductance of TMXYL molecules upon exposure to $\mathrm{TCNE}^{12}$ ). The other major difference is that a ChemDiode sensor element can be as small as a single sensor molecule or a single Au nanoparticle coated with sensor element molecules bridging the gap between source and drain 
contacts (see later discussion of nanoscale step junctions). Thus, a ChemDiode sensor is a true Nano-nose and many thousands of sensor elements can be accommodated on a single Si chip. A ChemDiode sensor one square micron in area contains approximately 40,000 sensor molecule/nanoparticle/sensor molecule elements. Thus, despite their small size, ChemDiode sensors are highly redundant and should have excellent long term stability. The huge surface-tovolume ratio of close-packed nanoparticle films ensures that ChemDiode sensors also have extremely fast response times relative to conventional chem-resistive devices.

In order to accomplish the goal of fabricating the ChemDiode platform we have developed a number of new technologies that should prove useful not only to the specific aims of the present project but also to achieving the more general goal of incorporating metal nanoparticles as contact elements to bridge molecular level events to mesoscopic and finally macroscopic electronic circuitry. As described in previous progress reports, we have developed:

1. A generic method by which large area, close-packed, monolayer films of molecularly protected nanoparticles (MPN's) (e.g. dodecanethiol coated nanocrystals of Au) can be self-assembled on a water surface. ${ }^{13}$ This method produces highly ordered monolayers of MPN's free of the microscopic holes and multilayer domains characteristic of monolayers produced by previous methods, Fig. 5.

2. A method by which these monolayer films can be transferred intact to solid substrates. In particular it is possible to transfer monolayers of MPN's to an elastomeric stamp (which has been previously patterned using a lithographically produced master) for subsequent printing on a target substrate. ${ }^{14}$ This method provides a way to fabricate laterally patterned, monolayer and multilayer films of MPN's that are close-packed arrays, Fig. 6.

3. A method by which molecular exchange between the molecules used to protect the nanoparticles during the self-assembly step and other molecules can take place in an organic solvent without dissolution of the self-assembled nanoparticle array or destruction of its close-packed structure. ${ }^{15}$

Electrode structures for the ChemDiode platform have been fabricated on oxidized $\mathrm{Si}$ substrates using photolithographic lift-off techniques (Fig. 7). These structures consist of $\mathrm{Au}$ pads $(100 \mathrm{~nm}$ thick deposited on a $10 \mathrm{~nm}$ thick Ti layer) that are separated by $100 \mu \mathrm{m}$. An interdigited pattern of Au fingers ( $30 \mathrm{~nm}$ thick deposited on a $5 \mathrm{~nm}$ thick Ti layer) is deposited between the pads. The fingers are $90 \mu \mathrm{m}$ in length and $2 \mu \mathrm{m}$ in width. Bilayer films of both $5 \mathrm{~nm}$ diameter and $10 \mathrm{~nm}$ diameter Au nanoparticles coated with dodecanethiol (DDT) have been printed on top of the electrode asssemblies to provide conduction channels for electron transport between the fingers. After the electrodes have been covered by a close-packed film of $\mathrm{Au}$ MPN's, the Si chip is immersed in a solvent containing the desired sensor molecule. The rate of exchange between DDT and the sensor molecule is monitored by transmission IR and/or UV-Vis spectroscopy using a similar nanoparticle film printed on a quartz substrate.
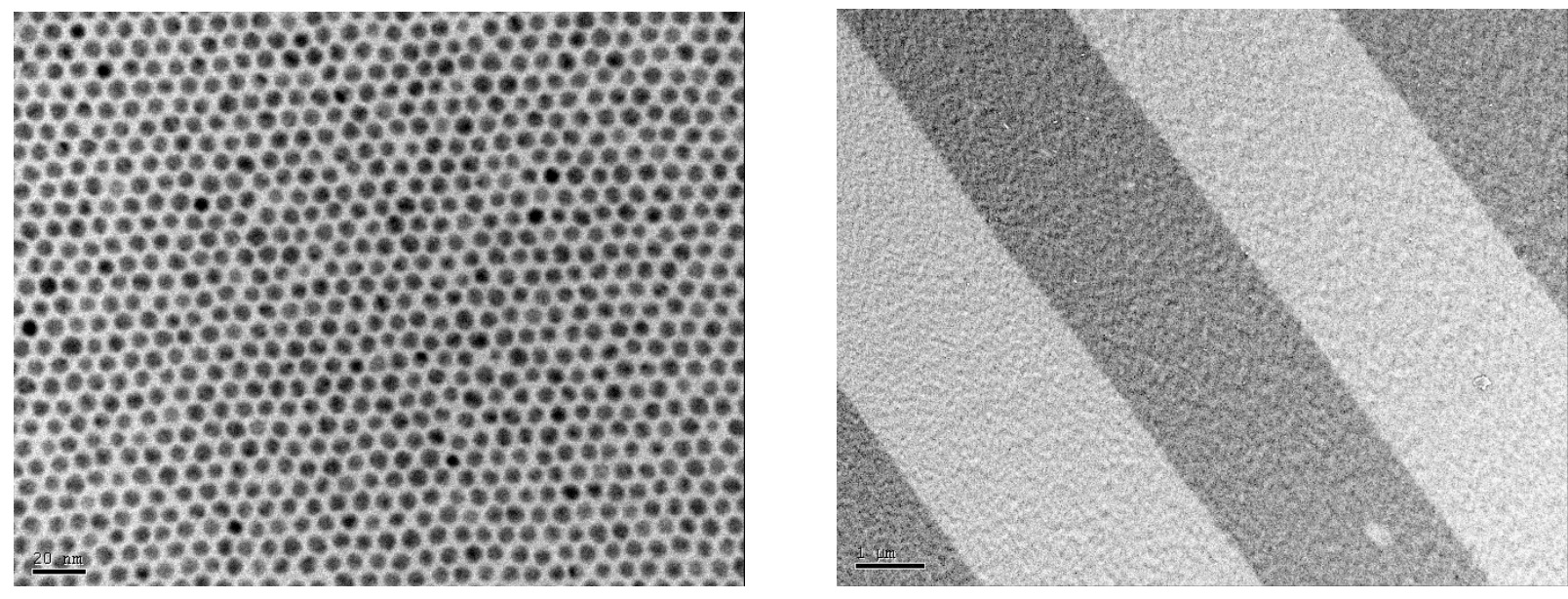
Figure 5. TEM micrograph of close-packed monolayer of $5 \mathrm{~nm}$ diameter $\mathrm{Au}$ nanoparticles coated with dodecanethiol molecules that was self-assembled on a water surface. ${ }^{13}$

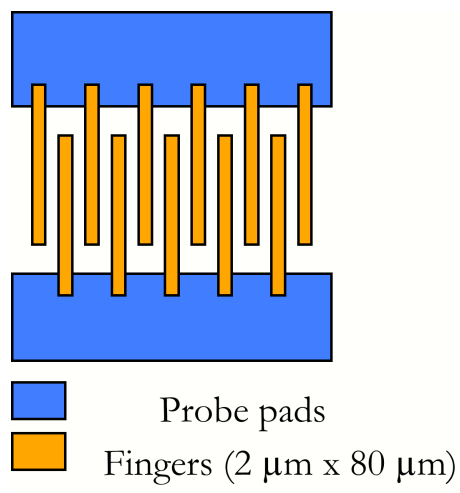

\section{Synthesis of Sensor Element Molecules}

Metallo-porphyrins are expected to be excellent sensor elements for small molecule sensing using either the ChemFET or the ChemDiode platform. Metallo-porphyrins bind many small gas molecules $\left(\mathrm{O}_{2}, \mathrm{CO}, \mathrm{NO}, \mathrm{SO}_{2}\right)$ to form adduct structures which differ significantly in their electronic structure compared to the unbound state. In addition, porphyrin molecules can be synthesized that contain many different transition metal atoms (e.g. Ti, $\mathrm{V}, \mathrm{Cr}, \mathrm{Mn}, \mathrm{Fe}, \mathrm{Ru}, \mathrm{Co}$, $\mathrm{Rh}, \mathrm{Ni}, \mathrm{Pd}, \mathrm{Cu}, \mathrm{Ag}, \mathrm{Zn}, \mathrm{Cd}, \mathrm{Hg}$ ) in a stable configuration. Figure 8 illustrates the molecular structure of two specific porphyrin molecules with protected thiol ligands that are suitable for binding to gold. Both of these molecules have been synthesized by the Kubiak group.

Porphyrins are known to undergo dramatic changes in their optical properties after adsorption of specific gas molecules. It is precisely this difference in electronic structure that can be used to detect the presence of various target species. The Kubiak group has adapted Lindsey's protected thiol porphyrin strategy for the synthesis of 5, 15- dithiol and 5, 10, 15, 20tetrathiol derivatives of tetraphenyl porphyrins (TPP's). ${ }^{24,25}$ They have shown that these molecules readily form SAMs on flat gold substrates and have demonstrated that TT-TPP molecules anchor themselves to atomically flat gold substrates by lying flat on the surface in a close-packed structure.
Figure 6. TEM micrograph of parallel lines of nanoparticles (5 $\mathrm{nm}$ diameter, coated with dodecanethiol) printed on a $\mathrm{Si}_{3} \mathrm{~N}_{4}$ membrane using a PDMS stamp. The bilayer lines are $2.5 \mu \mathrm{m}$ wide and are spaced $3.0 \mu \mathrm{m}$ apart. ${ }^{14}$
Figure 7. Electrode assembly for ChemDiode sensor. 


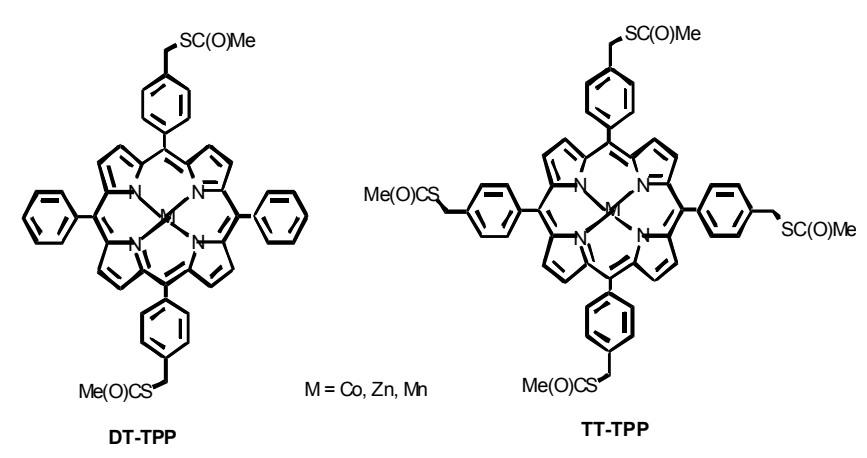

Figure 8. 5, 15-ditho-tetraphenyl porphryin (DT-TPP) and 5, 10, 15, 20-tetrathiotetraphenyl pophyrin (TT-TPP). Both are protected by acetyl thioester formation. Both are available in their metallated forms containing Co(II), Zn(II), or Mn(II).

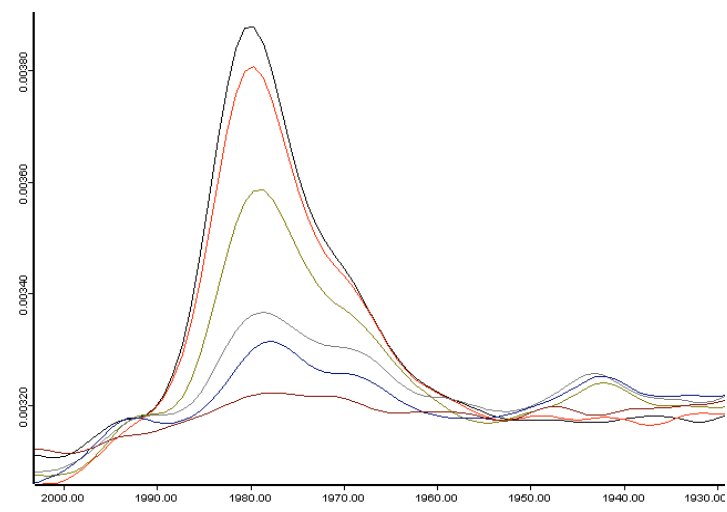

Figure 9.. Decreasing infrared $\mathrm{CO}$ absorption intensity with increased times of UV exposure with maximum intensity at $\sim 254 \mathrm{~nm}$. Time in seconds, $t=0,5,25,65$, $125,245$.

Another technique for forming a porphyrin SAM on gold is to first form a SAM of 4mercaptopyridine on the gold surface and then adsorb tetraphenyl porphyrin (TPP) onto this molecular layer. This technique promises to be an attractive method for incorporating metalloporphyrins into both ChemDiode and ChemFET devices. Using this technique a chemically reactive porphyrin SAM that reversibly binds $\mathrm{CO}$ was grown to calibrate our ability to detect the binding of small gas molecules on metallo-porphyrins. RuTPP(CO) (ruthenium tetraphenylporphyrin carbonyl) was self assembled onto a preformed SAM of 4mercaptopyridine on gold. Using surface IR methods the axially bound CO mode at $\sim 1980 \mathrm{~cm}^{-1}$ for the SAM can be observed. Importantly, the binding of CO to the RuTPP SAM is reversible. Thus, irradiating a RuTPP(CO) SAM with UV light causes the loss of CO (Fig. 9) and generation of a RuTPP SAM. Subsequent exposure of the RuTPP SAM to CO gas reforms the RuTPPCO SAM quantitatively. Surface potential changes of the RuTPP SAM upon CO binding are currently being measured, and ligand exchange reactions with other small molecules such as $\mathrm{NO}$ are being investigated.

Tetraphenyl porphyrins containing other metal centers which are chemically selective toward a range of target molecules are currently being synthesized. These molecules will be incorporated into the ChemDiode platform and tested as sensor elements for small molecules such as $\mathrm{CO}$ and $\mathrm{NO}$, and surface potential measurements will be made on SAM's of the molecules to evaluate their merit for ChemFET devices activated by molecular binding of small molecules.

\section{Introduction of Sensor Molecules into ChemDiode Devices}

Introducing a sensor molecule like a metallo-porphyrin into a close-packed array of dodecanethiol (DDT) coated Au nanoparticles so that this new molecule serves as the critical tunnel barrier between adjacent particles (Fig. 4) has proven to be extremely difficult. UV-Vis and IR spectra of monolayer and multilayer films of DDT coated Au nanoparticles indicate that although substantial molecular replacement takes place when the films are contacted with various linking molecules in solution, the complete removal of the DDT does not occur over a time scale of days. An example of this behavior is illustrated in Figure 10, which presents FTIR 
data for the replacement of DDT by xylyl dithiol (XYL) in a close-packed monolayer of $10 \mathrm{~nm}$ diameter $\mathrm{Au}$ particles supported on a quartz substrate. Dodecanethiol has signature IR absorption peaks at 2918-2920 $\mathrm{cm}^{-1}$ (for $\mathrm{CH}_{2}$ asymmetric stretch), $2854 \mathrm{~cm}^{-1}$ (for $\mathrm{CH}_{2}$ symmetric stretch), and $2964 \mathrm{~cm}^{-1}$ (for $\mathrm{CH}_{3}$ stretching). Thus, IR absorption can be used to monitor the number of $\mathrm{CH}_{2}$ and $\mathrm{CH}_{3}$ species in a nanoparticle film. Figure 10 shows that after an exposure to XYL in acetonitrile for 24 hours there is a continuing presence of DDT in the film. More importantly the electrical conductance of both monolayer and multilayer films of $\mathrm{Au}$ nanoparticles is not appreciably altered by contacting the films with XYL and various other linking molecules.

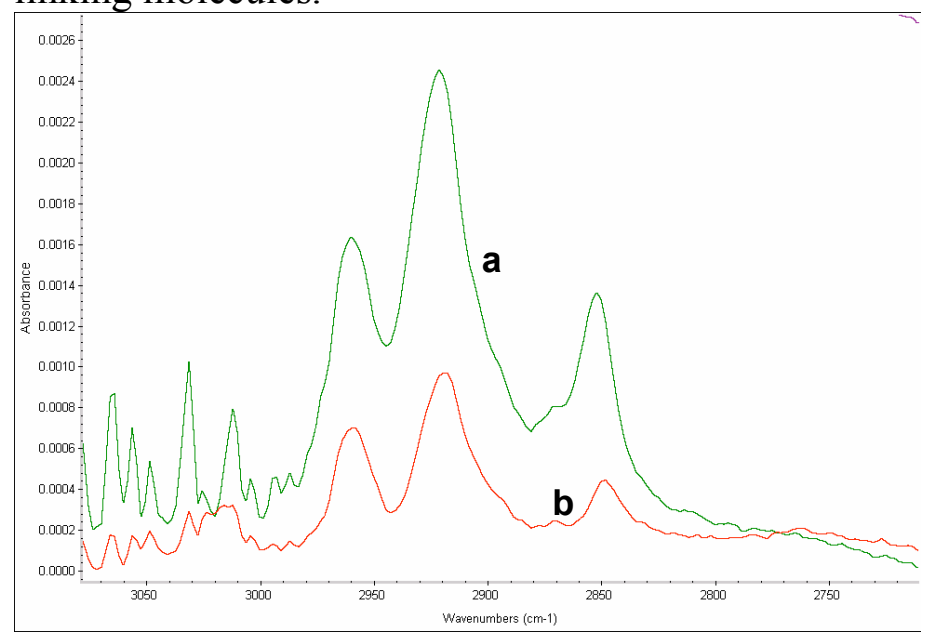

Figure 10. FTIR spectra for a closepacked monolayer of $10 \mathrm{~nm}$ diameter, DDT coated, $A u$ nanoparticles supported on a quartz substrate. a) film as formed, b) film after contact for 24 hours with a solution of XYL in acetonitrile.

Transmission electron microscopy (TEM) reveals that the particle-to-particle spacing in a close-packed monolayer of $\mathrm{Au}$ nanoparticles supported on an atomically flat $\mathrm{Si}_{3} \mathrm{~N}_{4}$ substrate is unchanged upon exposure to short replacement molecules such as XYL. Since electron tunnel resistance depends exponentially on distance, this TEM observation is consistent with the observation that the electrical resistance of the film does not change dramatically with molecular replacement, i.e. linking of the particles is not occurring even though molecular exchange takes place. This problem should not be present in the case of a multilayer film, however, as the vertical spacing between particle layers in the film can readily change even if the lateral spacing within the layers is constant. The electrical conductance data, however, do not confirm the hypothesis that as the DDT molecules coating the Au particles in a multilayer array are replaced by shorter molecules the distance between layers decreases and electrical tunnel resistance between layers in the film also decreases. The tentative conclusion is that much of the DDT remaining in a multilayer nanoparticle film after molecular exchange resides in the narrow regions between the aligned facets of adjacent particles. These residual DDT molecules keep the linking molecules from interconnecting the particles and keep the vertical spacing between particle layers from decreasing. Unless this DDT can be removed and replaced by the sensor molecule of interest, the electrical conductance of the film will not be affected by the presence of the sensor molecules and the film will not be chem-reistive.

It is possible to remove the DDT molecules separating adjacent particles in a multilayer film by heating the film, but this results in grain growth and destruction of the regular closepacked structure of the film. However, exposing a close-packed film of Au MPN's to ozone generated by UV radiation (e.g., at $253.7 \mathrm{~nm}$ and $184.9 \mathrm{~nm}$ wavelengths) in a flow of oxygen, i.e. low temperature UVO oxidation in a commercial UVO cleaning apparatus, is an effective 
way to remove DDT from the particles without adversely changing the structure of the film. The effect of UVO oxidation on a close-packed film of Au MPN's is: 1) to decrease the vertical height of the film without causing grain growth or changing the lateral spacing of the particles, 2) to dramatically increase the electrical conductance of the film, and 3) to improve the ability of various linking molecules to replace alkanethiol molecules coating the particles. TEM micrographs of a close-packed 4-layer film of $10 \mathrm{~nm}$ diameter $\mathrm{Au}$ MPN's supported on a $\mathrm{Si}_{3} \mathrm{~N}_{4}$ substrate show no apparent change in the structure of the film after 30 minutes of UVO oxidation. The rms surface roughness of the film, as measured by AFM, is of the order of $2 \mathrm{~nm}$ both before and after oxidation, however, the film thickness decreases from $\sim 60 \mathrm{~nm}$ to $\sim 20 \mathrm{~nm}$. The electrical sheet resistance of a similar 4-layer film supported on a $\mathrm{Si}$ substrate decreased from $2.4 \times 10^{9} \mathrm{ohms} / \mathrm{sq}$ to $91 \mathrm{ohms} / \mathrm{sq}$ or eight orders of magnitude when oxidized the same amount of time. UVO oxidation also has a dramatic effect on the rate of displacement of DDT by various linking molecules. Five minutes of UVO oxidation results in complete removal of the IR peaks associated with DDT when a monolayer such as the one described in Figure 10 is exposed for 30 minutes to XYL in acetonitrile.

The fact that with controlled UVO oxidation it may now be possible to exchange molecules like metallo-porphyrins into a multilayer, close-packed film of Au nanoparticles with some hope of introducing the desired molecule in the gap between particles in adjacent layers is encouraging. Experiments are underway to test whether exchanging either CoDT-TPP and CoTTP (anchored by 4-mercaptopyridine) into the current ChemDiode platform results in chemresistive behavior.

\section{Characterization of Molecular Conduction using Nanoscale Step Junctions}

The ultimate sensitivity of a ChemDiode device is achieved when the bias across each sensor molecule is large enough so that its tunnel resistance becomes non-linear. In an effort to achieve this we have fabricated sacrificial step junctions between metal electrodes that have gaps small enough to be bridged by individual sensor molecules and sensor molecule/nanoparticle/sensor molecule complexes. A cross-sectional schematic of such a sacrificial step junction is shown in Figure 11. The process for fabricating this nanoscale gap was described in detail in prior reports. Briefly, the process involves: i) the formation of a patterned metal contact, consisting of a thin layer of Au covered with a relatively thicker layer of a sacrificial material such as $\mathrm{Ti}$ or $\mathrm{Cr}$, ii) patterning of photoresist to define a second contact (overlapping with the first), iii) deposition of the second contact, using the edge of the first contact as a shadow mask to define the nanoscale gap between contacts, iv) lift-off of $2^{\text {nd }}$ contact metal, v) removal of sacrificial metal layers (by etching) to provide a planar contact structure. The development of this structure, and demonstration of it as a device to measure molecular conductivity, has recently been published. ${ }^{16}$
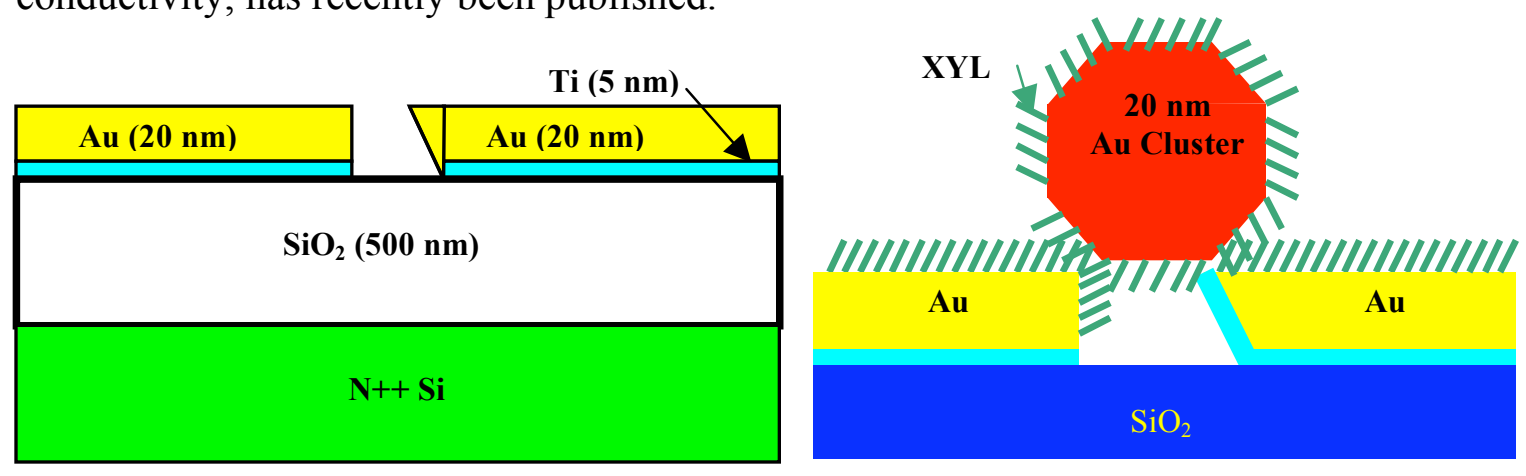
Figure 11. Schematic of the sacrificial step junction structure.
Figure 12. Schematic cross-section of device consisting of sacrificial step junction bridged with Au cluster and XYL

Imaging of the nanoscale gaps by field-emission scanning electron microscopy (FESEM) indicates that the average gap size along the width of the junction is in the range of 10-20 $\mathrm{nm}$. However, there is evidence that there are regions within some of the devices in which the local gap size is as small as the length of a small organic molecule. This is asserted by several observations. First, although it is not possible to resolve 1-2 $\mathrm{nm}$ a spacing using the FE-SEM, variations in the edge width of the gap are observed, which indicate that there are regions with gap width well below $10 \mathrm{~nm}$. Second, the measured conductivities of the devices implies gap spacings of approximately $1 \mathrm{~nm}$, based on an analysis of tunneling conduction through $\mathrm{metal} / \mathrm{vacuum} / \mathrm{metal}$ structures. ${ }^{26}$ Finally, observations made during studies using this device as a test structure for characterizing molecular conduction (described below) are also consistent with the realization of single-molecule scale gaps.

We have employed this structure (Fig. 11) and a structure in which the first electrode remains significantly thicker than the second electrode in studies of molecular conduction. Our first attempts involved exposing the structures to solutions containing various small organic molecules such as xylyl dithiol (XYL) and benzene dithiol. Although the yields were relatively low, significant increases in conductivity were observed in a number of devices following exposure to the molecular solutions. The interpretation of the increased conduction is that individual molecules have bonded across the gaps between the contacts. This implies that separations within the gap are sufficiently small in some regions to allow a short organic molecule to bridge the gap.

Although individual molecule studies can provide important insights into the conductance of organic molecules, an alternate approach provides higher yields, and allows development of approaches suitable for chemical sensing. The approach, illustrated in Fig. 12, involves the formation of a molecule/nanoparticle/molecule bridge, which can span contacts with gaps in the range of 10-20 nm. In this experiment, $20 \mathrm{~nm}$ diameter Au nanoparticles were employed, and the conducting molecule was xylyl dithiol. A self-assembled monolayer of XYL was deposited on the Au contacts, using standard solution techniques. The samples were then exposed to a colloidal suspension of citrate-stabilized Au particles, $20 \mathrm{~nm}$ in diameter. Following particle exposure, the electrical conductivity of the devices was measured, and a number of the devices were imaged using FE-SEM in order to quantify the number of particle-based bridges across the gaps.

Two FE-SEM images are shown in Fig. 13. The images are blurred somewhat because of carbon contamination, which might be due to the XYL SAM on the device surface. However, circular features with dimensions corresponding to the $20 \mathrm{~nm}$ diameters of the Au particles are clearly observed. This observation provides strong evidence that Au nanoparticles are deposited within the gap region. In some images, such as the one on the left in Fig. 13, there appear to be assemblies of Au particles within the gaps.
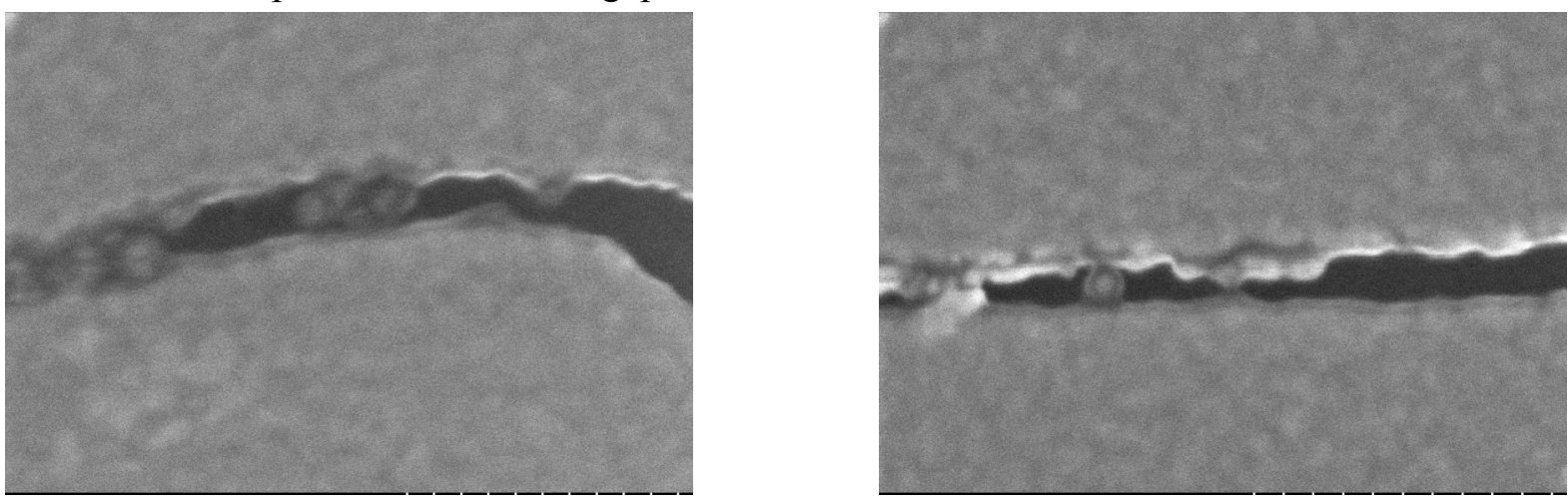
Figure 13. FE-SEM pictures from sacrificial step junction devices in which the gaps have been bridged with an assembly of XYL and Au clusters. In the first image, the bridge consists of a number of clusters in small arrays at center and left. In the second image, an individual cluster is observed to span the gap (mid-left). The other features are remnants of the metal edge from the first electrode; since the structure is three-dimensional, these remnants are not necessarily in the conduction path.
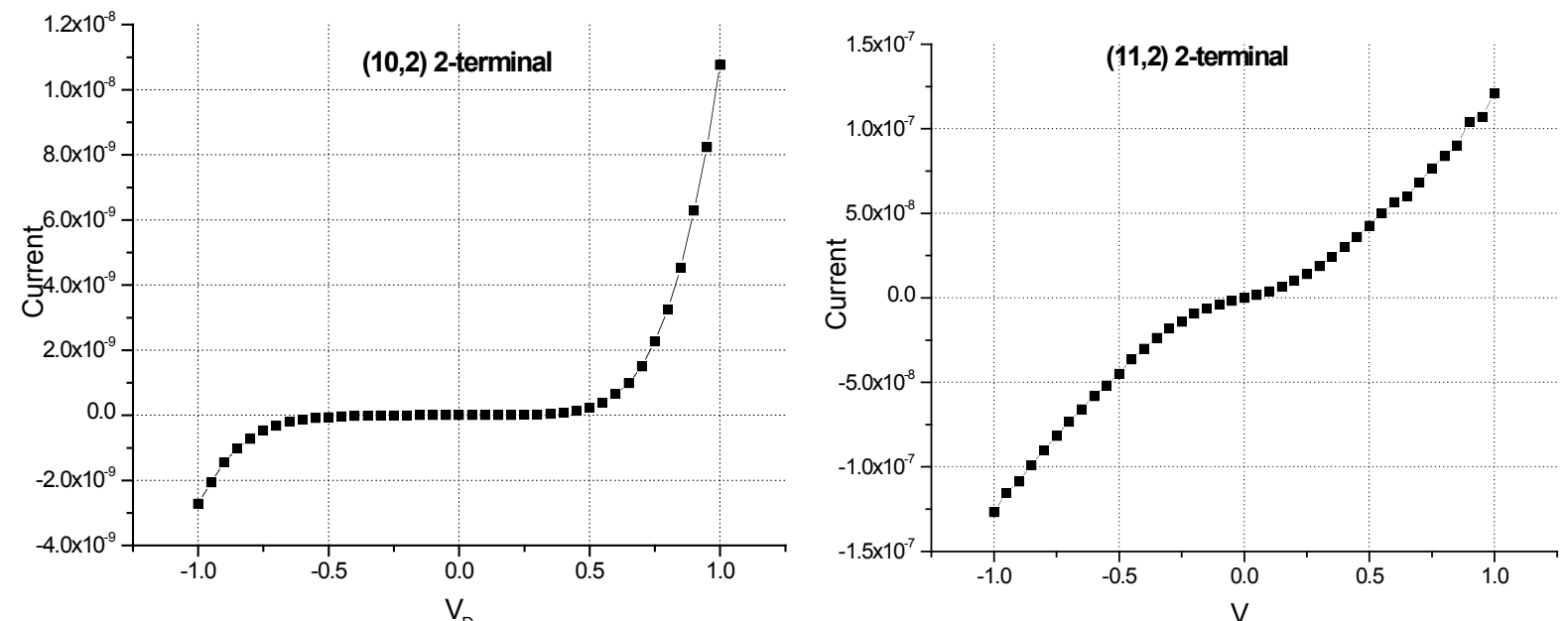

Figure 14. I-V curves from ${ }^{\mathrm{s}}$ acrificial step junction devices bridged by XYL coated $20 \mathrm{~nm}$ diameter Au particles

The molecule/nanoparticle/molecule devices exhibited significant enhancements in conductivity, with respect to the conductances measured i) before deposition of either molecules or nanoparticles and ii) after growth of the XYL SAM, but before deposition of the nanoparticles. This conductivity enhancement is believed to be due to conduction through the molecule/nanoparticle/molecule bridges. I-V curves observed for several of the devices are shown in Fig. 14. Note that the conduction is expected to be through a number of molecules, since each $20 \mathrm{~nm}$ diameter Au particle is large enough to accommodate $~ 100 \mathrm{XYL}$ molecules on a typical surface facet. With this taken into account, the observed conduction levels are in agreement with the expected conductance of XYL molecules.

\section{ChemDiode Platform Based on Nanoscale Step Junctions}

Based on the success achieved bridging the gap of a nanoscale step junction with molecule/nanoparticle/molecule complexes, we have experimented with a ChemDiode platform based on printing a close-packed array of Au MPN's across the nanoscale step junction gap. A FE-SEM image of such a device is shown in Fig. 15. The gap in this device is $\sim 20 \mathrm{~nm}$ (the dark band that runs vertically in the image at mid-left). A close-packed monolayer of $10 \mathrm{~nm}$ diameter DDT coated Au nanoparticles has been printed on top of the electrodes. 


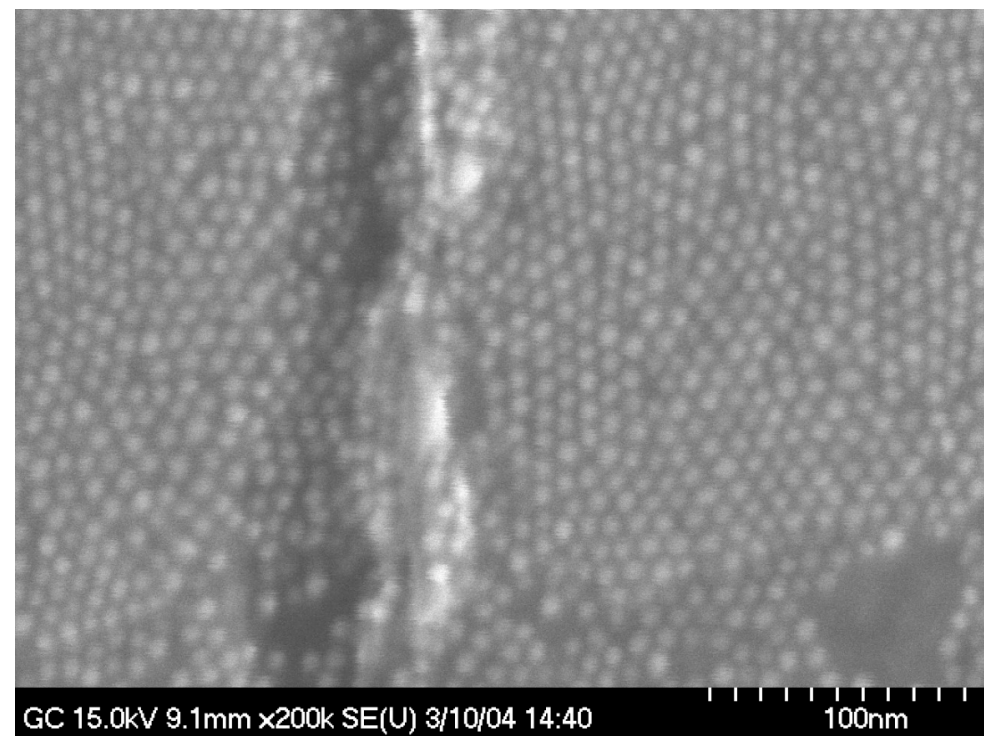

Figure 15. FE-SEM image of ChemDiode device based on sacrificial step junction covered by a close-packed monolayer of $\mathrm{Au}$ nanoparticles.

In order to render this structure chem-resistive the entire device was immersed for 12 hours in an equal molar mixture of FeTPP and 4-mercaptopyridine (1:1) in dichloromethane. After this treatment the I(V) characteristics of various junctions on the chip were measured in the presence and absence of CO. Although most junctions showed little if any chemical sensitivity, one junction exhibited chem-resitive behavior. The data for this experiment are shown in Figure 16. At a bias of 1 volt the device showed a change in conductivity of approximately $10 \%$ upon switching from pure $\mathrm{N}_{2}$ to a $10 \mathrm{ppm}$ mixture of $\mathrm{CO}$ in $\mathrm{N}_{2}$ at 310 torr and a corresponding smaller change when the switch occurred at 150 torr.

We are currently repeating and extending this experiment using multilayer nanoparticle films, using different sensor molecules, and using UVO oxidation treatment to improve on the replacement of DDT by the linking species. One fabrication problem that we face is the number of devices that are open circuits, i.e. have conductance values comparable to an untreated step junction. A possible explanation for this can be seen in the FE-SEM image in Figure 15. The spacing between the $10 \mathrm{~nm}$ diameter Au particles bridging the gap in the step junction is larger than the spacing for particles in the close-packed monolayer covering the surface of the electrodes. Even small increases in inter-particle spacing raise the electron tunneling resistance exponentially and make the possibility of linking adjacent particles with sensor molecules unlikely. One possible reason for this phenomenon is indicated by the lighter region to the right of the step junction gap, which is most probably due to a small ridge on the edge of the right electrode. Any elevation difference across the gap will tend to expand the distances between adjacent particles in the film. We are currently exploring several potential solutions to this problem: 1) trying to eliminate any ridge left by the lift-off process used to fabricate the sacrificial step junction, 2) transferring the Au nanoparticles as a sequential series of monolayers rather than as a single multilayer film, and 3) depositing a close-packed film of Au MPN's on the substrate first and then fabricating the sacrificial step junction on top of this film. 


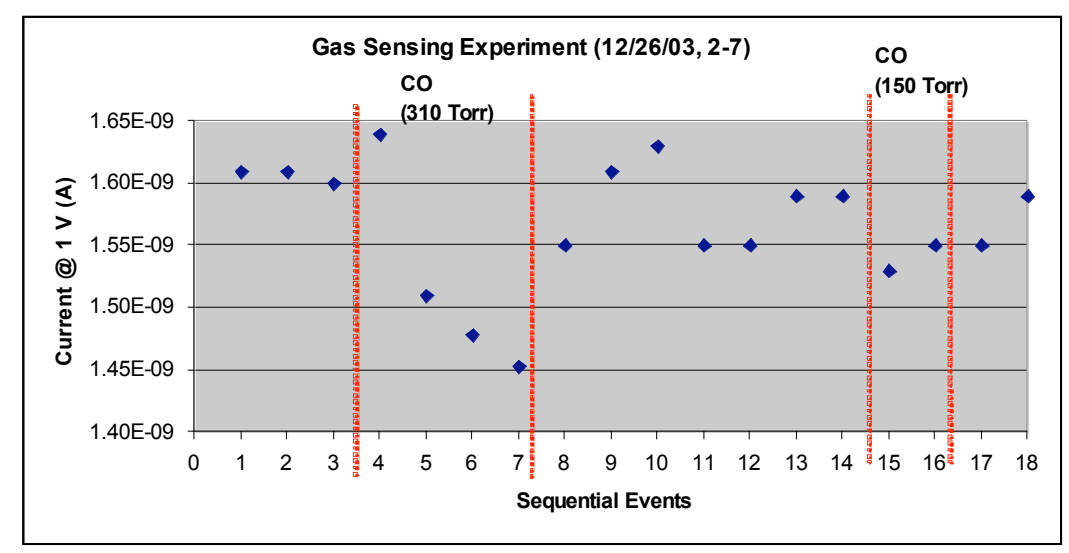

Figure 16. Response of sacrificial step junction 